Article

\title{
Whole-Genome Sequencing Analysis of Salmonella Enterica Serotype Enteritidis Isolated from Poultry Sources in South Korea, 2010-2017
}

\author{
Ji-Yeon Hyeon ${ }^{1,2}$, Shaoting Li ${ }^{1}$, David A. Mann ${ }^{1}$, Shaokang Zhang ${ }^{1}{ }^{(\mathbb{D}}$, Kyu-Jik Kim ${ }^{3}$, Dong-Hun Lee ${ }^{2}$, \\ Xiangyu Deng ${ }^{1}$ and Chang-Seon Song ${ }^{4, *(D)}$
}

check for updates

Citation: Hyeon, J.-Y.; Li, S.; Mann, D.A.; Zhang, S.; Kim, K.-J.; Lee, D.-H.; Deng, X.; Song, C.-S. Whole-Genome Sequencing Analysis of Salmonella Enterica Serotype Enteritidis Isolated from Poultry Sources in South Korea, 2010-2017. Pathogens 2021, 10, 45 https://doi.org/10.3390/ pathogens 10010045

Received: 10 December 2020

Accepted: 4 January 2021

Published: 7 January 2021

Publisher's Note: MDPI stays neutral with regard to jurisdictional clai$\mathrm{ms}$ in published maps and institutional affiliations.

Copyright: (C) 2021 by the authors. Licensee MDPI, Basel, Switzerland. This article is an open access article distributed under the terms and conditions of the Creative Commons Attribution (CC BY) license (https:// creativecommons.org/licenses/by/ $4.0 /)$.
1 Center for Food Safety, Department of Food Science and Technology, The University of Georgia, 1109 Experiment St, Griffin, GA 30223, USA; jiyeon.hyeon@uconn.edu (J.-Y.H.); shaoting.li25@uga.edu (S.L.); dmann29@uga.edu (D.A.M.); zskzsk@uga.edu (S.Z.); xdeng@uga.edu (X.D.)

2 Department of Pathobiology and Veterinary Science, The University of Connecticut, 61 N. Eagleville Road, Storrs, CT 06269, USA; dong-hun.lee@uconn.edu

3 KCAV Inc., KU Future Energy Research Center 202, 120 Neungdong-ro, Gwangjin-gu, Seoul 05029, Korea; zidanov31@gmail.com

4 Avian Disease Laboratory, College of Veterinary Medicine, Konkuk University, 120 Neungdong-ro, Gwangjin-gu, Seoul 05029, Korea

* Correspondence: songcs@konkuk.ac.kr; Tel.: +82-2-3437-1940

\begin{abstract}
Salmonella enterica subsp. enterica serotype Enteritidis (SE) is recognized as a major cause of human salmonellosis worldwide, and most human salmonellosis is due to the consumption of contaminated poultry meats and poultry byproducts. Whole-genome sequencing (data were obtained from 96 SE isolates from poultry sources, including an integrated broiler supply chain, farms, slaughterhouses, chicken transporting trucks, and retail chicken meats in South Korea during 2010-2017. Antimicrobial resistance and virulence genes were investigated using WGS data, and the phylogenetic relationship of the isolates was analyzed using single-nucleotide polymorphism (SNP) typing and core genome multilocus sequence typing (cgMLST). All isolates carried aminoglycoside resistance genes, aac $\left(6^{\prime}\right)-I a a$, and 56 isolates carried multiple antimicrobial resistance genes. The most frequent virulence gene profile, pef-fim-sop-inv.-org-sip-spa-sif-fli-flg-hil-ssa-sse-prg-pag-spv, was found in 90 isolates. The SNP analysis provided a higher resolution than the cgMLST analysis, but the cgMLST analysis was highly congruent with the SNP analysis. The phylogenetic results suggested the presence of resident SE within the facility of processing plants, environments of slaughterhouses, and the integrated broiler supply chain, and the phylogenetically related isolates were found in retail meats. In addition, the SE isolates from different origins showed close genetic relationships indicating that these strains may have originated from a common source. This study could be valuable reference data for future traceback investigations in South Korea.
\end{abstract}

Keywords: Salmonella enteritidis; poultry; antibiotic resistance gene; virulence gene; SNP analysis; cgMLST analysis

\section{Introduction}

Nontyphoidal Salmonella enterica (S. enterica) is one of the most prevalent foodborne pathogens in the world [1,2]. Among Salmonella serotypes, S. enterica serotype Enteritidis (S. Enteritidis, SE) is the most common Salmonella serotype associated with foodborne illnesses in the United States and Europe, and its common sources are poultry and poultry products [3]. In South Korea, S. enterica was the second most common pathogen associated with foodborne illnesses over the past 15 years, according to the foodborne outbreak surveillance system of Korea's Food and Drug Administration (https: / / www.foodsafetykorea.go.kr/portal/healthyfoodlife/foodPoisoningStat.do). To identify 
infection sources and transmission routes, the epidemiological association between poultry sources and human disease has been investigated using different molecular typing methods [4-7].

Recently, whole-genome sequencing (WGS) has been frequently implemented in routine surveillance of foodborne pathogens as well as foodborne disease outbreak investigation [2,7-11]. WGS enables serotyping, antimicrobial resistance, virulence profiling, and subtyping in a single WGS workflow [12]. In addition, the application of WGS in laboratory surveillance provides higher resolution and precision than pulsed-field gel electrophoresis (PFGE), which had been the gold standard for PulseNet [12]. Therefore, PulseNet has worked toward implementing WGS into routine public health surveillance since 2014, and PFGE has been phased out and replaced with WGS as PulseNet's primary surveillance tool in 2019 [13]. Currently, there are several studies that applied WGS to molecular characterization and phylogenetic analysis of the Salmonella spp. isolated from various sources $[2,5,7,14-20]$. However, only a few studies using WGS analysis for Salmonella spp. in South Korea have been reported [21-23], and WGS is not yet routinely used as a tool for genetic characterization of SE in the food supply chain in South Korea.

There are two analytical approaches of WGS-based subtyping: base-by-base (singlenucleotide polymorphism [SNP] analysis) or gene-to-gene (multilocus sequence typing [MLST]) [24]. For the base-by-base approach, an SNP is a nucleotide difference at a specific position in the genome of a test strain relative to the sequence of a reference strain and occurs because of a genetic mutation event [24]. The MLST approach considers allelic variants in a defined set of gene loci [19]. This approach is very flexible because the number and the nature of the genes assessed can be tailored to any given situation and genomes in question [24]. Core genome MLST (cgMLST) balances the number of loci used in a scheme by including those loci present in the majority of isolates (ranging from $95 \%$ to $99 \%$ ) in a given species [17].

Given the significance and repeated outbreaks of SE in South Korea together with the limited availability of WGS data, we sequenced the whole genome of $96 \mathrm{SE}$ isolates isolated from poultry sources in South Korea during 2010-2017, including retail chicken meats, farms, slaughterhouses, an integrated broiler supply chain, and chicken transporting trucks. The presence of antimicrobial resistance genes, chromosomal mutation, and virulence genes in the SE isolates was investigated using WGS data. Then we conducted a phylogenetic analysis using SNP and cgMLST analysis to investigate the genetic relatedness of SE isolates sequenced in this study.

\section{Results}

2.1. Detection of Antimicrobial Resistance Genes and Mutations in the gyrA, gyrB, parC and parE Genes

The WGS data were used to detect the presence of antimicrobial resistance genes in the 96 isolates, and a total of 12 antimicrobial resistance patterns were observed (Table 1 and Supplementary Table S1). The most frequent antimicrobial resistance pattern was pattern 1 $(n=27,28.1 \%)$, followed by pattern $10(n=19,19.8 \%)$ and pattern $5(n=15,15.6 \%)$. Among 96 isolates, 56 isolates $(58.3 \%)$ carried multiple antimicrobial resistance genes (Table 1$)$, and one isolate from the chicken transporting truck (KR105) contained 5 antimicrobial resistance genes (Figure 1). A total of 12 antibiotic resistance genes were identified and are described in detail below, according to the different antimicrobial classes (Table 1).

\subsubsection{Aminoglycoside Resistance Genes}

All 96 isolates carried aminoglycoside resistance genes, aac ( $\left.6^{\prime}\right)$-Iaa, and four other different genes including aph(6)-Id $(n=53,55.2 \%)$, aph (3")-Ib $(n=53,55.2 \%)$, aac(3)-Iid $(n=17,17.7 \%)$, and $\operatorname{aph}\left(3^{\prime}\right)-\operatorname{Ia}(n=16,16.7 \%)$ were also detected. 
Table 1. Antimicrobial genes detected in the 96 Salmonella enterica subsp. enterica serotype enteritidis (SE) isolates in this study.

\begin{tabular}{|c|c|c|c|c|c|c|c|c|}
\hline \multirow{2}{*}{ Pattern } & \multicolumn{6}{|c|}{ Antimicrobial Genes (Identity, \%) } & \multirow{2}{*}{$\begin{array}{l}\text { Chromosome } \\
\text { Mutations }\end{array}$} & \multirow{2}{*}{$\begin{array}{l}\text { Number of } \\
\text { Isolates }(\%)\end{array}$} \\
\hline & Aminoglycoside & Sulfonamide & Trimethoprim & Beta-Lactam & Tetracycline & Phenicol & & \\
\hline Pattern 1 & $\begin{array}{c}\text { aac(6')-Iaa (96.4), aph(3")-Ib (100), } \\
\text { aph(6)-Id (99.76-100) }\end{array}$ & sul2 (100) & & $\begin{array}{c}\text { blaTEM-1B } \\
(99.9-100)\end{array}$ & $\operatorname{tet}(A)(99.9-100)$ & & gyrA p.D87N & $\begin{array}{c}27 \\
(28.1)\end{array}$ \\
\hline Pattern 2 & $\begin{array}{c}a a c\left(6^{\prime}\right)-\operatorname{Iaa}(96.4), \operatorname{aph}\left(3^{\prime \prime}\right)-\operatorname{Ib}(100) \\
\operatorname{aph}(6)-\operatorname{Id}(99.76-100)\end{array}$ & sul2 (100) & & $\begin{array}{c}\text { blaTEM-1B } \\
(99.9-100)\end{array}$ & $\operatorname{tet}(A)(99.9-100)$ & & - & $\begin{array}{c}4 \\
(4.2)\end{array}$ \\
\hline Pattern 3 & $\begin{array}{c}a a c\left(6^{\prime}\right)-I a a(96.4), \operatorname{aph}\left(3^{\prime \prime}\right)-I b(100) \\
\operatorname{aph}(6)-\operatorname{Id}(100)\end{array}$ & sul2 (100) & & blaTEM-1B (100) & & catA2 (96.1) & gyrA p.D87G & $\begin{array}{c}5 \\
(5.2)\end{array}$ \\
\hline Pattern 4 & $\begin{array}{c}\text { aac(6')-Iaa (96.4), aph(3")-Ib (100), } \\
\text { aph(6)-Id (99.8) }\end{array}$ & sul2 (100) & & blaTEM-1B (100) & & & - & $\begin{array}{c}1 \\
(1.0) \\
\end{array}$ \\
\hline Pattern 5 & $\begin{array}{c}a a c(3)-\operatorname{Iid}(99.9) \\
\operatorname{aac}\left(6^{\prime}\right)-\operatorname{Iaa}(96.4), \operatorname{aph}\left(3^{\prime \prime}\right)-\operatorname{Ib}(99.9) \\
\operatorname{aph}\left(3^{\prime}\right)-\operatorname{Ia}(100), \operatorname{aph}(6)-\operatorname{Id}(100)\end{array}$ & sul2 (100) & & blaCTX-M-15 (100) & $\operatorname{tet}(A)(97.3-100)$ & & gyrA p.D87N & $\begin{array}{c}15 \\
(15.6)\end{array}$ \\
\hline Pattern 6 & $\begin{array}{c}a a c(3)-\operatorname{Iid}(99.9) \\
\operatorname{aac}\left(6^{\prime}\right)-\operatorname{Iaa}(96.4), \operatorname{aph}\left(3^{\prime \prime}\right)-\operatorname{Ib}(99.9) \\
\operatorname{aph}\left(3^{\prime}\right)-\operatorname{Ia}(100), \operatorname{aph}(6)-\operatorname{Id}(100)\end{array}$ & sul2 (100) & & blaCTX-M-15 (100) & $\operatorname{tet}(A)(97.3-100)$ & & - & $\begin{array}{c}1 \\
(1.0)\end{array}$ \\
\hline Pattern 8 & $a a c\left(6^{\prime}\right)-\operatorname{Iaa}(96.4)$ & Sul1 (100) & $d f r A 1(95.4)$ & blaTEM-1B (100) & $\operatorname{tet}(A)(98.8)$ & & gyrA p.D87G & $\begin{array}{c}1 \\
(1.0)\end{array}$ \\
\hline Pattern 9 & $a a c\left(6^{\prime}\right)-I a a(96.4)$ & & & & & & gyrA p.D87N & $\begin{array}{c}7 \\
(7.3)\end{array}$ \\
\hline Pattern 10 & $a a c\left(6^{\prime}\right)-I a a(96.4)$ & & & & & & gyrA p.D87G & $\begin{array}{c}19 \\
(19.8)\end{array}$ \\
\hline Pattern 11 & $a a c\left(6^{\prime}\right)-I a a(96.4)$ & & & & & & - & $\begin{array}{c}14 \\
(14.6)\end{array}$ \\
\hline Pattern 12 & $\begin{array}{l}\operatorname{aac}(3)-\operatorname{Iid}(99.9) \\
\operatorname{aac}\left(6^{\prime}\right)-\operatorname{Iaa}(96.4)\end{array}$ & & & $\begin{array}{c}\text { blaCTX-M-15 } \\
\quad(99.7)\end{array}$ & & & gyrA p.D87N & $\begin{array}{c}1 \\
(1.0)\end{array}$ \\
\hline $\begin{array}{l}\text { Number of } \\
\text { isolates (\%) }\end{array}$ & $\begin{array}{c}96 \\
(100)\end{array}$ & $\begin{array}{c}55 \\
(57.3)\end{array}$ & $\begin{array}{c}2 \\
(2.1)\end{array}$ & $\begin{array}{c}56 \\
(58.3)\end{array}$ & $\begin{array}{c}48 \\
(50 \%)\end{array}$ & $\begin{array}{c}1 \\
(1.0)\end{array}$ & $\begin{array}{c}75 \\
(78.1)\end{array}$ & \\
\hline
\end{tabular}




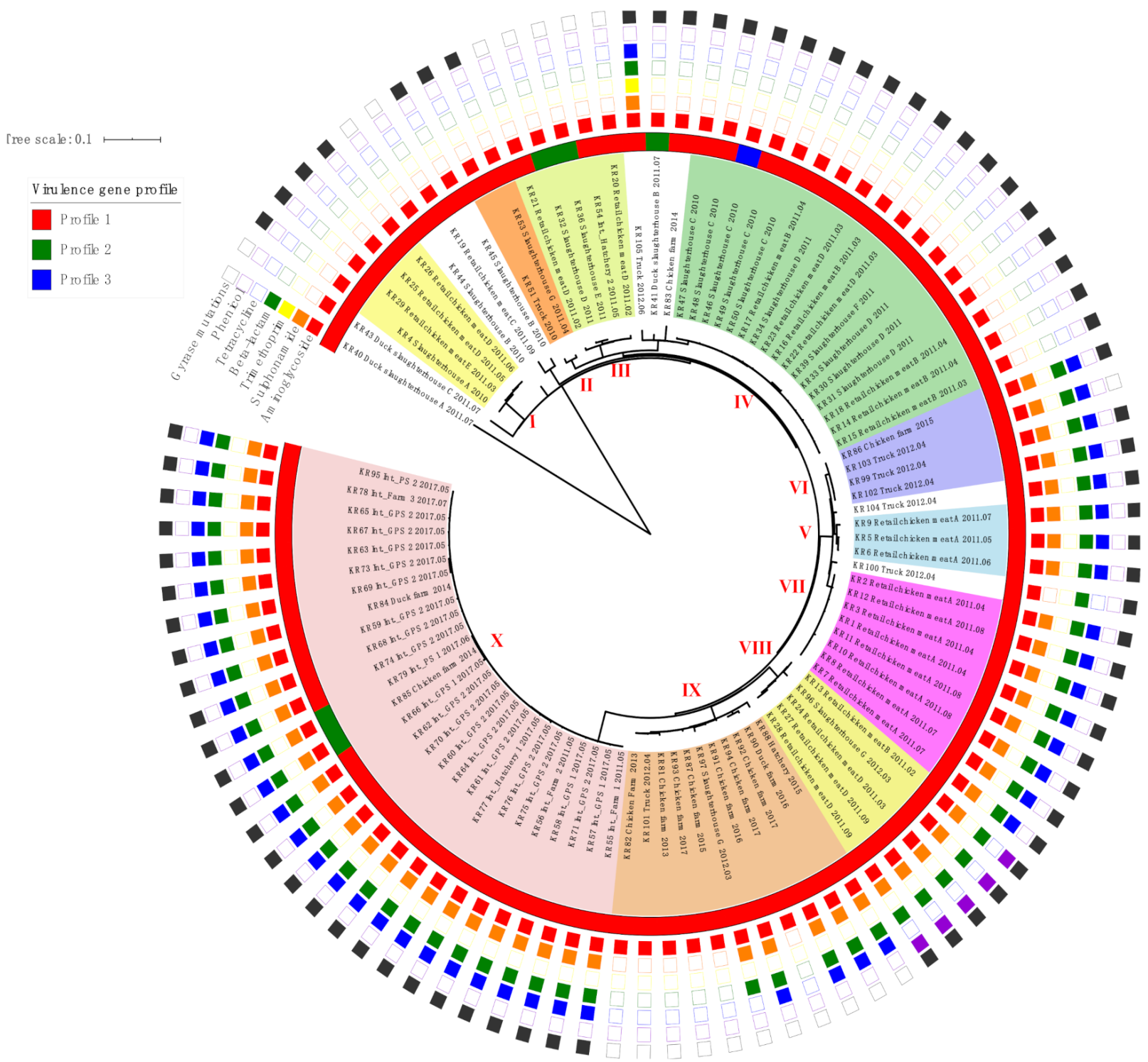

Figure 1. Phylogenetic single-nucleotide polymorphism (SNP) tree, antibiotic resistance patterns and virulence gene profiles of 96 SE isolates visualized using interactive Tree Of Life version 5 (iTOLv5) (https://itol.embl.de/). The SE isolates belonging to SNP groups (I to X) are highlighted with different colors, and the presence of antimicrobial genes and virulence gene profiles of each SE isolates are indicated. Int., integrated broiler supply chain; GPS, grandparent stock; PS, parent stock.

\subsubsection{Sulfonamide and Trimethoprim Resistance Genes}

Two sulfonamide resistance genes were found in 55 (57.3\%) isolates including sul2 ( $n=54,56.2 \%)$ and $\operatorname{sul} 1(n=1,1.0 \%)$, and only one trimethoprim resistance gene, $d f r A 1$, was detected in two (2.1\%) isolates.

\subsubsection{Beta-Lactam Resistance Genes}

Two beta-lactams resistance genes were detected in $56(58.3 \%)$ isolates including bla $_{\text {TEM-1B }}(n=39,40.6 \%)$ and bla $_{\text {CTX-M-15 }}(n=17,17.7 \%)$.

\subsubsection{Tetracycline Resistance Genes}

Only one tetracycline resistance gene, tet $(A)$, was detected in 48 (50\%) isolates. 


\subsubsection{Phenicol Resistance Genes}

One phenicol resistance gene, cat A2, was found in five (5.2\%) isolates.

\subsubsection{Detection of Mutations in the gyrA, gyrB, parC and parE Genes}

A total of 75 (78.1\%) out of 96 isolates presented point mutation in the gyrA gene, with all being resistant to nalidixic acid and ciprofloxacin (Figure 1, Supplementary Table S1). The nonsynonymous point mutation in the gyrA gene included: aspartate/glycine, D87G in 25 isolates (26.0\%); aspartate/asparagine, D87N in 50 isolates (52.1\%). None of the isolates had point mutation $\mathrm{n}$ the gyrB, parC and parE genes (data not shown).

\subsection{Virulence Gene Profiles}

The genomes SE isolates tested were investigated for 21 virulence genes. From the results of the virulence gene investigation, three virulence gene profiles were established (Figure 1, Table 2). The most frequently detected profile was profile 1 (pef-fim-sop-inv.-orgsip-spa-sif-fli-flg-hil-ssa-sse-pro-pag-spv) $(n=90,93.8 \%)$ followed by profile 2 (fim-sop-inv.-orgsip-spa-sif-fli-flg-hil-ssa-sse-prg-pag) $(n=5,5.2 \%)$ and profile 3 (pef-fim-sop-inv-org-sip-spa-siffli-flg-hil-ssa-sse-prg-pag) $(n=1,1.0 \%)$. All isolates carried fim, sop, inv, org, sip, spa, sif, fli, flg, hil, ssa, sse, prg, and pag, and none of the strains carried sef, sfm, spr, stn, and bss (Table 2).

Table 2. Virulence genes detected in the 96 SE isolates in this study.

\begin{tabular}{|c|c|c|c|c|c|c|c|}
\hline \multirow[b]{2}{*}{ Profile } & \multicolumn{6}{|c|}{ Virulence Genes } & \multirow[b]{2}{*}{$\begin{array}{l}\text { Number of } \\
\text { Isolates(\%) }\end{array}$} \\
\hline & $\begin{array}{l}\text { Adhesion } \\
\text { Effectors } \\
\text { (sef, pef, sfm } \\
\text { and fim) }\end{array}$ & $\begin{array}{c}\text { Invasion } \\
\text { Effectors } \\
\text { (sop, inv., org, } \\
\text { sip, spa, sif, fli, } \\
\text { flg, hil and spr) }\end{array}$ & $\begin{array}{l}\text { Host Cell } \\
\text { Survival Effectors } \\
\text { (ssa, sse, prg } \\
\text { and pag) }\end{array}$ & $\begin{array}{l}\text { Virulence } \\
\text { Plasmid } \\
\text { Gene } \\
(s p v)\end{array}$ & $\begin{array}{l}\text { Enterotoxin- } \\
\text { Producing } \\
\text { Gene } \\
\text { (stn) }\end{array}$ & $\begin{array}{l}\text { Biofilm } \\
\text { Regulator } \\
\quad(b s s)\end{array}$ & \\
\hline Profile 1 & Pef, fim & $\begin{array}{l}\text { sop, inv., org, sip, } \\
\text { spa, sif, fli, flg, hil }\end{array}$ & ssa, sse, prg, pag & spv & - & - & $\begin{array}{c}90 \\
(93.8)\end{array}$ \\
\hline Profile 2 & Fim & $\begin{array}{l}\text { sop, inv., org, sip, } \\
\text { spa, sif, fli, flg, hil }\end{array}$ & ssa, sse, prg, pag & - & - & - & $\begin{array}{c}5 \\
(5.2)\end{array}$ \\
\hline Profile 3 & Pef, fim & $\begin{array}{l}\text { sop, inv., org, sip, } \\
\text { spa, sif, fli, flg, hil }\end{array}$ & ssa, sse, prg, pag & - & - & - & $\begin{array}{c}1 \\
(1.0)\end{array}$ \\
\hline
\end{tabular}

\subsection{Phylogenetic Analysis of SE Isolates}

The 96 SE genomes were assessed for their phylogenetic relationships using both SNP and cgMLST analysis (Figures 1-3). According to the framework for interpreting SNP analysis provided by the FDA-CFSAN [25], we considered the isolates are closely related, increasing the likelihood that they arose from the same source when (i) there are 20 or fewer SNPs and (ii) the phylogenetic analysis shows a monophyletic relationship with bootstrap support of 0.90 or higher. The SE genomes were grouped into 10 different groups (I to $\mathrm{X}$ ), and ten singleton genomes were not clustered into any major group (Figures 1 and 2). The SE isolates in the identical phylogenetic groups showed similar antibiotic resistance patterns (Figure 1).

In SNP phylogeny (Figure 2), group I consisted of four closely related isolates from the retail chicken meats of brand $\mathrm{D}(n=2)$ and $\mathrm{E}(n=1)$ and the slaughterhouse $\mathrm{A}(n=1)$ (median 2.5 SNPs, range 0-5 SNPs). Two isolates from the slaughterhouse $\mathrm{G}$ and the truck clustered in the group II (median 5 SNPs, range 0-11 SNPs). Group III included five isolates from the retail chicken meats of brand $\mathrm{D}(n=2)$, slaughterhouse $\mathrm{D}(n=1)$ and $\mathrm{E}(n=1)$, and the hatchery 2 of the integrated broiler supply chain $(n=1)$ (median 5 SNPs, range 0-9 SNPs). In group IV, five of six isolates from the retail chicken meats of brand B were clustered together with the isolates from the retail chicken meats of brand $\mathrm{D}(n=2)$ and the slaughterhouse $\mathrm{C}(n=5), \mathrm{D}(n=4)$, and F $(n=1)$ (median 2.5 SNPs, range 0-6 SNPs). In group $\mathrm{V}$, three isolates from the retail chicken meats of brand A clustered together (median 2 SNPs, range 0-10 SNPs). Group VI contained four isolates from the trucks $(n=3)$ and 
a chicken farm $(n=1)$ (median 5 SNPs, range 0-11 SNPs), and eight SE isolates from the retail chicken meats of brand A clustered together in the group VII (median 1 SNPs, range 0-5 SNPs). Group VIII included five isolates from the retail meat chicken meats of brand B $(n=1)$ and $\mathrm{D}(n=3)$ and the slaughterhouse $\mathrm{G}(n=1)$ (median 4 SNPs, range $0-14$ SNPs). In group IX, seven isolates from chicken farms were clustered together with the isolates from the duck farm $(n=1)$, the slaughterhouse $\mathrm{G}(n=1)$, and the truck $(n=1)$ (median 8 SNPs, range 0-18 SNPs). Group $X$ included 27 isolates from the integrated broiler supply chain $(n=25)$, duck farm $(n=1)$ and chicken farm $(n=1)$ (median 2 SNPs, range 0-8 SNPs). Among the 26 isolates from the integrated broiler supply chain, 25 isolates were clustered together into group $\mathrm{X}$.

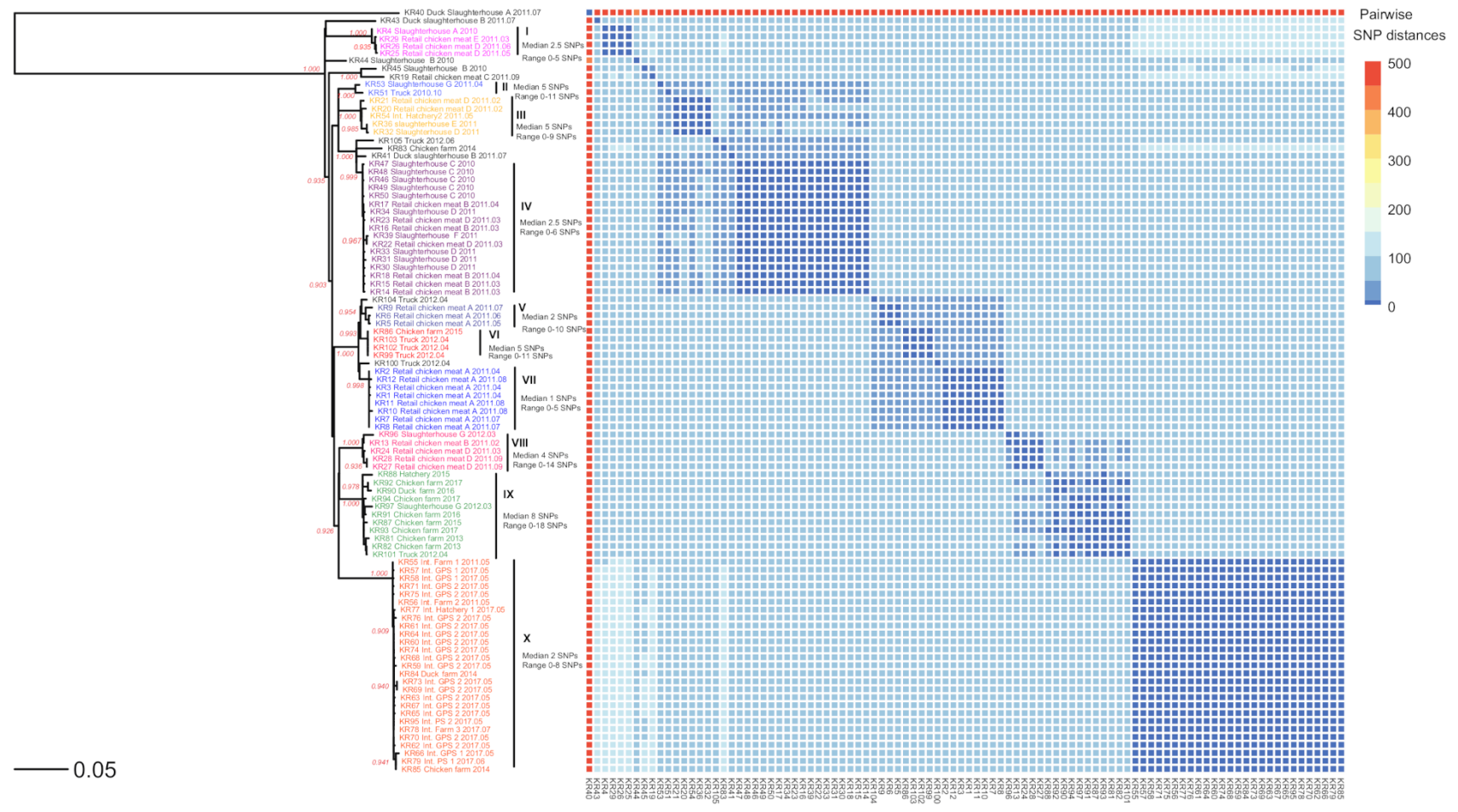

Figure 2. Phylogenetic analysis of 96 SE isolates using SNP analysis. The phylogeny was rooted at the midpoint. A total of 1034 SNP sites were identified. The colored SE isolates were grouped into 10 groups (I to X). Pairwise SNP distances (0 to 492 SNPs) are indicated by the heat map. Colors in the heat map indicate the numbers of pairwise SNP distances between isolates, with blue being the lowest and light orange being the largest. The scale bar measures the number of substitutions per site. Ratios on certain tree branches indicate branch support by the default approximate likelihood ratio test (aLRT) in PhyML. Only support greater than 0.9 is shown. Int., integrated broiler supply chain; GPS, grandparent stock; PS, parent stock.

The results of cgMLST analysis showed that the clustering of the isolates resembled that of the SNP analysis, such as the SNP group I, III, VIII, IX and X (Figure 3). However, there were some differences in clustering from the SNP analysis. The SE isolates in the SNP group V, VI and VII were grouped into two clusters, and the SNP group II and IV were clustered together in cgMLST. 


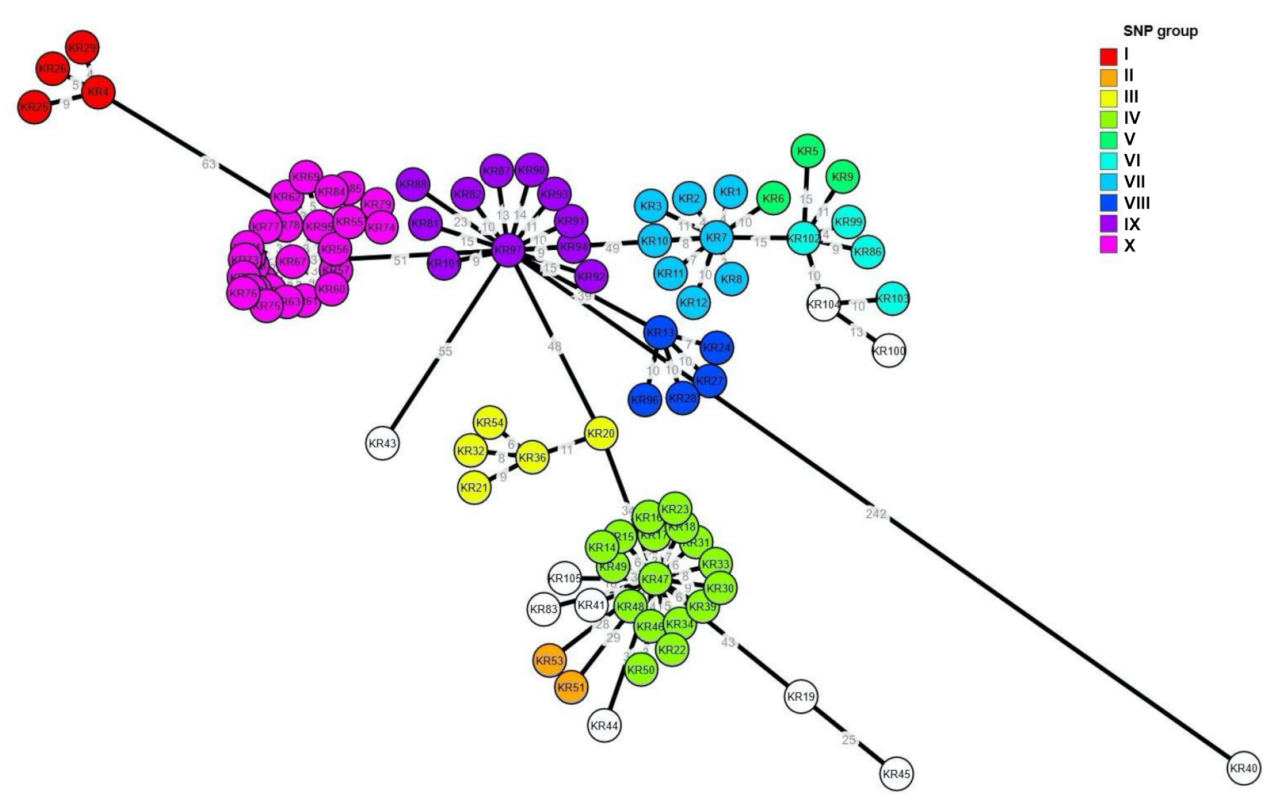

Figure 3. Core genome multilocus sequence typing (cgMLST)-based minimum spanning tree [created with cgMLST Finder 1.1(https:/ / cge.cbs.dtu.dk/services/CSIPhylogeny/) and GrapeTree version 1.5.0 (https: / / github.com/achtman-lab/GrapeTree)] of the 96 SE isolates of this study. The colors of the nodes indicate the groups of SNP analysis (I to X), and the numbers at the branches indicate the allelic differences to the nearest neighbor.

\section{Discussion}

WGS analysis has been used to investigate genetic characteristics, and phylogenetic relationships among Salmonella isolates from different origins, such as humans, food, animals or environmental samples in previous studies $[6,14,17,25]$. In this study, the genomes of SE isolates from poultry sources in broiler production chains in South Korea were sequenced to evaluate their antimicrobial resistance gene pattern, virulence gene profile and phylogenetic diversity.

Several previous studies reported that phenotypic resistance and genotypic resistance correlated highly in Salmonella spp. [15,17,26,27], but some of the discrepancies in aminoglycosides and beta-lactams were noted $[14,15,17,27]$. Genotypic antimicrobial resistance found in this study agreed with the phenotypic antimicrobial resistance of Salmonella spp. from poultry sources, in that there was high resistance to streptomycin, nalidixic acid, sulfisoxazole, and cephalothin, found in previous studies from South Korea [26-28]. The finding of multidrug-resistant Salmonella spp. in the Korean poultry production food supply chain is concerning due to the potential transmission to humans. High resistance to antimicrobials and multidrug resistance of Salmonella spp. from poultry sources were also reported in other countries $[29,30]$. For example, resistance to erythromycin, penicillin, and vancomycin have been reported as the most common resistance profile of Salmonella spp. from raw chicken meat in Malaysia [30] and poultry and poultry environments in India [31]. In Zhou et al.'s study [32], among 146 SEisolates obtained from retail chicken products in Shanghai, China, $50.70 \%$ of the isolates were resistant to ampicillin, $49.32 \%$ to sulfisoxazole, $17.12 \%$ to tetracycline, and $15.75 \%$ to doxycycline, and $30(20.55 \%)$ isolates were resistant to three or more antimicrobials. In Canada, more than $43 \%$ of 193 Salmonella isolates recovered from commercial farms were simultaneously resistant to ampicillin, amoxicillin-clavulanic acid, ceftiofur, cefoxitin, and ceftriaxone [33].

A total of 90 SE isolates (93.8\%) from different poultry sources showed the same virulence gene profile involved in adhesion, invasion, cell survival, and virulence plasmid genes. However, neither enterotoxin-producing genes nor biofilm regulator genes were detected in the strains tested. In previous studies [13,17,34], Salmonella isolates recovered from the animal, food, and/or environmental sources contained the same virulence genes 
as carried by human clinical isolates, and these findings highlight the pathogenic potential of Salmonella causing disease in humans via contaminated food.

Most SE isolates from the same origin phylogenetically clustered together even though sampling dates and sampling locations of the samples were different in this study. For example, the SE isolates from the retail chicken meats of brand A collected in April, July and August in 2011 clustered together in the group VII, and the SE isolates from the evisceration rooms, chilling rooms, and carcasses in slaughterhouse $C$ all clustered together in the group IV (Supplementary Table S1, Figures 1 and 2). Together, these data suggest the presence of a resident SE within the processing plant and slaughterhouse environments.

SE isolates from the different stages in the integrated broiler supply chain, such as grandparent stocks, parent stocks, hatcheries, and farms clustered together in the group $\mathrm{X}$ (Supplementary Table S1, Figures 1 and 2). Interestingly, these SE isolates were collected in 2011 and 2017, and SE was not detected in the integrated broiler supply chain between 2012 and 2016 (data not shown). It remains uncertain how this SE strain was undetected and perpetuated in the chain during 2012-2016 and reappeared in 2017. Our SNP analysis data suggest that the SE strain persisted in the integrated broiler supply chain without an introduction of a new SE strain and evolved at a low nucleotide substitution rate of approximately 0.33 nucleotides per year compared to the evolutionary rate (1.01 nucleotide substitutions per year) estimated by a previous study of S. enterica [5]. Taken together, we assume that the SE strain was maintained at a low level in the integrated broiler supply chain but was not re-isolated until it reemerged in 2017.

The SE isolates from different origins showed close genetic relationships, and epidemiological or traceback evidence was provided to establish the connection between these SE isolates in the identical groups. In group IX, two SE isolates (KR 97 and KR 101) collected in the same year, 2012, from the slaughterhouse G and the truck which transported culled chickens to the slaughterhouse $G$ were grouped together (Supplementary Table S1, Figures 1 and 2). Therefore, the hypothesis that these isolates originated from the same source is supported by the epidemiological evidence and the phylogenetic analysis results.

Consistent with previous studies $[17,19]$, both SNP and cgMLST analysis were highly congruent, but the SNP analysis provided a higher resolution than the cgMLST analysis in the phylogenetic analysis. The differences between SNP and cgMLST analysis may be due to a combination of these factors, i) a cgMLST scheme do not include intergenic regions, ii) only one allelic change are counted when multiple nucleotide changes within the same gene can be found, and iii) short insertions or deletions in the core genes are ignored by the SNP analysis, but captured by cgMLST analysis [17]. In addition, unlike an SNP analysis by the CFSAN SNP pipeline, no guideline for interpretation of the cgMLST results has been established; how many allelic differences are necessary to conclude that two strains are from the different origin [19].

\section{Materials and Methods}

\subsection{Bacterial Isolates}

The 96 SE isolates sequenced for this study were selected from SE isolates collected from poultry sources in South Korea between 2010 and 2017. The 83 isolates were isolated by Konkuk University in Seoul, South Korea, during 2010-2017, and 13 isolates were kindly provided by the Korean National Animal and Plant Quarantine Agency (APQA) in Gimcheon-si, South Korea (Supplementary Table S1).

For Salmonella isolation, $200 \mathrm{~mL}$ of buffered peptone water (BPW) (Difco Laboratories, Detroit, MI, USA) was added to the swab samples in sterile plastic jars with caps and incubated at $37^{\circ} \mathrm{C}$ for $18-24 \mathrm{~h}$. For the carcass rinse samples, rinsed BPW was transferred to a sterile plastic jar with a cap and incubated at $37^{\circ} \mathrm{C}$ for $18-24 \mathrm{~h}$ for pre-enrichment. For selective enrichment, $100 \mu \mathrm{L}$ of enriched BPW broth culture was transferred to $9.9 \mathrm{~mL}$ of Rappaport-Vassiliadis broth (Difco Laboratories) and incubated at $42{ }^{\circ} \mathrm{C}$ for $24-48 \mathrm{~h}$. Following incubation, the Rappaport-Vassiliadis broth enrichment cultures were streaked onto xylose lysine deoxycholate agar (Difco Laboratories) and brilliant green agar (Difco Labo- 
ratories), and the plates were incubated overnight at $37^{\circ} \mathrm{C}$. Two presumptive Salmonella colonies from each sample were transferred to MacConkey agar (Difco Laboratories). Colonies with a positive result were confirmed by Salmonella-specific PCR, as previously described [35]. Serotyping was performed using antisera (Difco Laboratories) in agglutination tests on the basis of somatic $\mathrm{O}$ antigen and phase 1 and phase 2 flagella antigens according to the Kauffmann-White scheme.

Those isolates included 28 isolates from retail chicken meats of five different brands (A to E), 17 isolates from six chicken slaughterhouses (A to $G$ ), four isolates from two duck slaughterhouses, 37 isolates from hatcheries and chicken farms, two isolates from duck farms, and eight isolates from chicken transporting trucks. Among 96 SE isolates, 26 SE isolates were from the integrated broiler supply chain A; two grandparent stocks $(n=19)$, two hatcheries $(n=2)$, two parent stocks $(n=2)$, and two broiler farms $(n=3)$.

The slaughterhouse isolates originated from carcasses and environmental samples (chiller water and wall and floor of the bleeding room, evisceration room, chilling room, selection room, and processing rooms). The hatchery and farm isolates were from environmental samples (litter, dust, and drag swabs), carcasses, livers, feces, cloaca swabs, eggshells, and fluff.

\subsection{Genome Sequencing}

Sample DNA concentrations were determined using a Qubit BR dsDNA assay kit (Thermo Fisher Scientific Inc., Waltham, MA, USA). Concentrations were diluted to $0.2 \mathrm{ng} / \mu \mathrm{L}$, and libraries were prepared following the Illumina Nextera XT DNA library prep kit (Illumina, Inc., San diego, CA, USA) reference guide with the following exceptions. Forty microliters of PCR product transferred to a new MIDI plate, $20 \mu \mathrm{L}$ of AMPure XP beads (Beckman Coulter, Brea, CA, USA) were added to each well and incubated at room temperature for $5 \mathrm{~min}$ without shaking. After $80 \%$ ethanol washes, beads were allowed to air dry for $12 \mathrm{~min}$. Then beads were resuspended in $53 \mu \mathrm{L}$ of RSB and incubated at room temperature for 2 min without shaking.

The concentration of sample libraries was determined using the Qubit dsDNA HS assay kit (Thermo Fisher Scientific Inc., Waltham, MA, USA), and libraries were diluted to a $2 \mathrm{nM}$ concentration and combined in equal volumes to form the pooled library. The pooled library was denatured to obtain a 10 pM final library following the Illumina Denature and Dilute Libraries Guide-Protocol A. Six hundred $\mu$ l of the denatured 10 pM library was loaded into the MiSeq reagent cartridge.

\subsection{Sequencing Reads Trimming, Filtering, and Classification}

Trimmomatic [36] was used to remove low-quality reads. The leading three and the trailing three nucleotides were removed from the reads, and a 4-nucleotide sliding window was used to remove nucleotides from the $3^{\prime}$ end when the average Phred score dropped below 20. Additionally, reads shorter than $50 \mathrm{bp}$ were discarded after trimming.

\subsection{De Novo Assembly and Serotyping}

The trimmed reads were assembled using SPAdes [34] with the "-careful" option. QUAST [37] was used to evaluate the quality of draft genome assemblies and determine the N50 value for each assembly. SeqSero [38] was used to predict Salmonella serotype from raw sequencing reads.

\subsection{Antibiotic Resistance Analysis}

The presence of acquired antimicrobial resistance genes as well as chromosomal mutations in the gyr $A, \operatorname{gyr} B, \operatorname{par} C$, and parE genes were determined using assembled genomes and ResFinder 3.2 (Center for Genomic Epidemiology, https: / / cge.cbs.dtu.dk/ services/ResFinder/) with settings of a threshold of $90 \%$, and a minimum length of $60 \%$ [39]. 


\subsection{Virulence Genes Investigation}

All genomes were annotated using the RASTtk algorithm [40] using the PATRIC v3.6.8. annotation server with default parameters (https:/ / patricbrc.org/app/Annotation) [41]. Protein annotations, including those for proteins specifically asserted to be involved in virulence factors, were downloaded from the PATRIC workspace and used for subsequent analyses. Twenty-one virulence genes were investigated in this study, including adhesion effectors (sef, pef, sfm and fim), invasion effectors (sop, inv., org, sip, spa, sif, fli, flg, hil and spr), host cell survival effectors (ssa, sse, prg and pag), virulence plasmid gene (spv), enterotoxinproducing gene $(s t n)$ and biofilm regulator (bss) [42].

\subsection{Phylogenetic Analysis}

High-quality SNPs were identified using CFSAN SNP Pipeline v2.0.2 using default quality filters [43]. Specifically, minimum base quality was 15; minimum mapping quality was 30; minimum fraction of reads for SNP calls was 0.9. Genomes of SE strain P125109 (NCBI reference sequence NC_011294.1) were used as reference genomes for reads mapping. Default pipeline settings were used for MiSeq reads. An SNP matrix and aligned SNPs were produced by the CFSAN SNP pipeline. Finally, maximum-likelihood (ML) phylogenetic trees were constructed using PhyML [44]. In addition, the phylogenetic data were visualized with antibiotic resistance patterns and virulence gene profiles using interactive Tree Of Life version 5 (iTOLv5) (https:/ /itol.embl.de/).

For cgMLST, raw sequence data files of the isolates were uploaded to cgMLST Finder 1.1 of the Center for Genomic Epidemiology (https://cge.cbs.dtu.dk/services/ cgMLSTFinder/) using the scheme developed by EnteroBase Salmonella database (https: / / enterobase.warwick.aC.uk) that considers a total of 3002 loci. The minimum spanning tree based on allelic profiles was created using GrapeTree version 1.5.0 [45].

\section{Conclusions}

In conclusion, this study demonstrated the application of WGS to predict antimicrobial resistance, detect virulence genes, and assess the phylogenetic relationship among SE isolates from different poultry sources in South Korea. In addition, both SNP and cgMLST analyses were highly congruent. Our results demonstrated that WGS is a powerful tool for investigating the epidemiology of Salmonella in the poultry industry, from farm to retail chicken meat, in conjunction with epidemiology and traceback findings. In addition, this study could be valuable reference data for future traceback investigations in the poultry industry in South Korea.

Supplementary Materials: The following are available online at https:/ / www.mdpi.com/2076-081 $7 / 10 / 1 / 45 /$ s1, Supplementary Table S1. Whole-genome sequencing characterization of 96 SE isolates used in this study.

Author Contributions: Conceptualization, D.-H.L., and C.-S.S.; methodology, J.-Y.H., D.A.M., S.L., S.Z. and X.D.; resources, K.-J.K. and C.-S.S.; data curation, J.-Y.H., D.A.M., K.-J.K., S.L., S.Z., and X.D.; writing—original draft preparation, J.-Y.H.; writing—review and editing, D.-H.L. and X.D.; visualization, J.-Y.H., S.L., and S.Z. supervision, C.-S.S.; funding acquisition, C.-S.S. All authors have read and agreed to the published version of the manuscript.

Funding: This work was supported by the Korea Institute of Planning and Evaluation for Technology in Food, Agriculture, Forestry (IPET) through the Animal Disease Management Technology Development Program, Funded by the Ministry of Agriculture, Food and Rural Affairs (MAFRA) (119055-02).

Institutional Review Board Statement: Not applicable.

Informed Consent Statement: Not applicable.

Data Availability Statement: The WGS data presented in this study are openly available in NCBI Bioproject database, Accession number (PRJNA623127). 
Acknowledgments: The authors would like to thank the Korean National Animal and Plant Quarantine Agency for kindly providing the bacterial strains. This paper was written as part of Konkuk University's research support program for its faculty on sabbatical leave in 2017.

Conflicts of Interest: The authors declare no conflict of interest.

\section{References}

1. Scallan, E.; Hoekstra, R.M.; Angulo, F.J.; Tauxe, R.V.; Widdowson, M.A.; Roy, S.L.; Jones, J.L.; Griffin, P.M. Foodborne illness acquired in the United States-major pathogens. Emerg. Infect. Dis. 2011, 17, 7-15.

2. Leekitcharoenphon, P.; Hendriksen, R.S.; Le Hello, S.; Weill, F.X.; Baggesen, D.L.; Jun, S.R.; Ussery, D.W.; Lund, O.; Crook, D.W.; Wilson, D.J.; et al. Global Genomic Epidemiology of Salmonella enterica Serovar Typhimurium DT104. Appl. Environ. Microbiol. 2016, 82, 2516-2526.

3. Ferrari, R.G.; Rosario, D.K.A.; Cunha-Neto, A.; Mano, S.B.; Figueiredo, E.E.S.; Conte-Junior, C.A. Worldwide Epidemiology of Salmonella Serovars in Animal-Based Foods: A Meta-analysis. Appl. Environ. Microbiol. 2019, 85, e00591-19.

4. Choi, S.W.; Ha, J.S.; Kim, B.Y.; Lee, D.H.; Park, J.K.; Youn, H.N.; Hong, Y.H.; Lee, S.B.; Lee, J.B.; Park, S.Y.; et al. Prevalence and characterization of Salmonella species in entire steps of a single integrated broiler supply chain in Korea. Poult. Sci. 2014, 93, 1251-1257.

5. $\quad$ Deng, X.; Desai, P.T.; den Bakker, H.C.; Mikoleit, M.; Tolar, B.; Trees, E.; Hendriksen, R.S.; Frye, J.G.; Porwollik, S.; Weimer, B.C.; et al. Genomic epidemiology of Salmonella enterica serotype Enteritidis based on population structure of prevalent lineages. Emerg. Infect. Dis. 2014, 20, 1481-1489.

6. Lee, D.H.; Hyeon, J.Y.; Kim, J.; Kim, J.S.; Kim, S.J.; Jeon, S.E.; Choi, S.W.; Hong, W.T.; Song, C.S.; Lee, S.W. Close genetic relationship between Salmonella enterica serovar Enteritidis isolated from patients with diarrhoea and poultry in the Republic of Korea. Clin. Microbiol. Infect. 2015, 21, e68-e70.

7. Toro, M.; Retamal, P.; Ayers, S.; Barreto, M.; Allard, M.; Brown, E.W.; Gonzalez-Escalona, N. Whole-Genome Sequencing Analysis of Salmonella enterica Serovar Enteritidis Isolates in Chile Provides Insights into Possible Transmission between Gulls, Poultry, and Humans. Appl. Environ. Microbiol. 2016, 82, 6223-6232.

8. Lienau, E.K.; Strain, E.; Wang, C.; Zheng, J.; Ottesen, A.R.; Keys, C.E.; Hammack, T.S.; Musser, S.M.; Brown, E.W.; Allard, M.W.; et al. Identification of a salmonellosis outbreak by means of molecular sequencing. N. Engl. J. Med. 2011, 364, 981-982.

9. Eppinger, M.; Mammel, M.K.; Leclerc, J.E.; Ravel, J.; Cebula, T.A. Genomic anatomy of Escherichia coli O157:H7 outbreaks. Proc. Natl. Acad. Sci. USA 2011, 108, 20142-20147.

10. Rasko, D.A.; Webster, D.R.; Sahl, J.W.; Bashir, A.; Boisen, N.; Scheutz, F.; Paxinos, E.E.; Sebra, R.; Chin, C.S.; Iliopoulos, D.; et al. Origins of the E. coli strain causing an outbreak of hemolytic-uremic syndrome in Germany. N. Engl. J. Med. 2011, 365, 709-717.

11. Deng, X.; den Bakker, H.C.; Hendriksen, R.S. Genomic Epidemiology: Whole-Genome-Sequencing-Powered Surveillance and Outbreak Investigation of Foodborne Bacterial Pathogens. Annu. Rev. Food Sci. Technol. 2016, 7, 353-374.

12. Carleton, H.A.; Gerner-Smidt, P. Whole-genome sequencing is taking over foodborne disease surveillance. Microbe Mag. 2016, 11, 311-317.

13. Tolar, B.; Joseph, L.A.; Schroeder, M.N.; Stroika, S.; Ribot, E.M.; Hise, K.B.; Gerner-Smidt, P. An Overview of PulseNet USA Databases. Foodborne Pathog. Dis. 2019, 16, 457-462.

14. Almeida, F.; Medeiros, M.I.; Kich, J.D.; Falcao, J.P. Virulence-associated genes, antimicrobial resistance and molecular typing of Salmonella Typhimurium strains isolated from swine from 2000 to 2012 in Brazil. J. Appl. Microbiol. 2016, 120, 1677-1690.

15. Almeida, F.; Seribelli, A.A.; Medeiros, M.I.C.; Rodrigues, D.D.P.; de MelloVarani, A.; Luo, Y.; Allard, M.W.; Falcao, J.P. Phylogenetic and antimicrobial resistance gene analysis of Salmonella Typhimurium strains isolated in Brazil by whole genome sequencing. PLOS ONE 2018, 13, e0201882.

16. McDermott, P.F.; Tyson, G.H.; Kabera, C.; Chen, Y.; Li, C.; Folster, J.P.; Ayers, S.L.; Lam, C.; Tate, H.P.; Zhao, S. Whole-Genome Sequencing for Detecting Antimicrobial Resistance in Nontyphoidal Salmonella. Antimicrob. Agents Chemother 2016, 60, 5515-5520.

17. Pearce, M.E.; Alikhan, N.F.; Dallman, T.J.; Zhou, Z.; Grant, K.; Maiden, M.C.J. Comparative analysis of core genome MLST and SNP typing within a European Salmonella serovar Enteritidis outbreak. Int. J. Food Microbiol. 2018, $274,1-11$.

18. Pornsukarom, S.; van Vliet, A.H.M.; Thakur, S. Whole genome sequencing analysis of multiple Salmonella serovars provides insights into phylogenetic relatedness, antimicrobial resistance, and virulence markers across humans, food animals and agriculture environmental sources. BMC Genom. 2018, 19, 801.

19. Simon, S.; Trost, E.; Bender, J.; Fuchs, S.; Malorny, B.; Rabsch, W.; Prager, R.; Tietze, E.; Flieger, A. Evaluation of WGS based approaches for investigating a food-borne outbreak caused by Salmonella enterica serovar Derby in Germany. Food Microbiol. 2018, 71, 46-54.

20. Tay, M.Y.F.; Pathirage, S.; Chandrasekaran, L.; Wickramasuriya, U.; Sadeepanie, N.; Waidyarathna, K.D.K.; Liyanage, L.D.C.; Seow, K.L.G.; Hendriksen, R.S.; Takeuchi, M.T.; et al. Whole-Genome Sequencing Analysis of Nontyphoidal Salmonella enterica of Chicken Meat and Human Origin Under Surveillance in Sri Lanka. Foodborne Pathog. Dis. 2019, 16, 531-537.

21. Kim, S.; Kim, E.; Park, S.; Hahn, T.W.; Yoon, H. Genomic Approaches for Understanding the Characteristics of Salmonella enterica subsp. enterica Serovar Typhimurium ST1120, Isolated from Swine Feces in Korea. J. Microbiol. Biotech. 2017, $27,1983-1993$.

22. Park, A.K.; Shin, E.; Kim, S.; Park, J.; Jeong, H.J.; Chun, J.H.; Hwang, K.J.; Kim, J. The Traveler-associated High-level Ciprofloxacinresistant Salmonella enterica Serovar Kentucky in the Republic of Korea. J. Global Antimicrobial. Resist. 2019. [CrossRef] 
23. Woojung, L.; Sewook, P.; Ran Hee, Y.; In-Sun, J.; Hyo Sun, K.; Soon Han, K. Complete genome sequence of Salmonella Enteritidis MFDS1004839 isolated from food. Korean J. Microbiol. 2018, 54, 164-166.

24. Brown, E.; Dessai, U.; McGarry, S.; Gerner-Smidt, P. Use of Whole-Genome Sequencing for Food Safety and Public Health in the United States. Foodborne Pathog. Dis. 2019, 16, 441-450.

25. Pightling, A.W.; Pettengill, J.B.; Luo, Y.; Baugher, J.D.; Rand, H.; Strain, E. Interpreting Whole-Genome Sequence Analyses of Foodborne Bacteria for Regulatory Applications and Outbreak Investigations. Front. Microbiol. 2018, 9, 1482.

26. Kim, M.S.; Lim, T.H.; Jang, J.H.; Lee, D.H.; Kim, B.Y.; Kwon, J.H.; Choi, S.W.; Noh, J.Y.; Hong, Y.H.; Lee, S.B.; et al. Prevalence and antimicrobial resistance of Salmonella species isolated from chicken meats produced by different integrated broiler operations in Korea. Poult Sci. 2012, 91, 2370-2375.

27. Lee, S.K.; Choi, D.; Kim, H.S.; Kim, D.H.; Seo, K.H. Prevalence, Seasonal Occurrence, and Antimicrobial Resistance of Salmonella spp. Isolates Recovered from Chicken Carcasses Sampled at Major Poultry Processing Plants of South Korea. Foodborne Pathog. Dis. 2016, 13, 544-550.

28. Yoon, R.H.; Cha, S.Y.; Wei, B.; Roh, J.H.; Seo, H.S.; Oh, J.Y.; Jang, H.K. Prevalence of Salmonella isolates and antimicrobial resistance in poultry meat from South Korea. J. Food Prot. 2014, 77, 1579-1582.

29. Cardoso, M.O.; Ribeiro, A.R.; Santos, L.R.D.; Pilotto, F.; Moraes, H.L.S.d.; Salle, C.T.P.; Rocha, S.L.d.S.; Nascimento, V.P.d. Antibiotic resistance in Salmonella Enteritidis isolated from broiler carcasses. Brazilian J. Microbiol. 2006, 37, 368-371.

30. Thung, T.Y.; Mahyudin, N.A.; Basri, D.F.; Wan Mohamed Radzi, C.W.; Nakaguchi, Y.; Nishibuchi, M.; Radu, S. Prevalence and antibiotic resistance of Salmonella Enteritidis and Salmonella Typhimurium in raw chicken meat at retail markets in Malaysia. Poult Sci. 2016, 95, 1888-1893.

31. Singh, R.; Yadav, A.S.; Tripathi, V.; Singh, R.P. Antimicrobial resistance profile of Salmonella present in poultry and poultry environment in north India. Food Control. 2013, 33, 545-548.

32. Zhou, X.; Xu, L.; Xu, X.; Zhu, Y.; Suo, Y.; Shi, C.; Shi, X. Antimicrobial Resistance and Molecular Characterization of Salmonella enterica Serovar Enteritidis from Retail Chicken Products in Shanghai, China. Foodborne Pathog. Dis. 2018, 15, $346-352$.

33. Diarra, M.S.; Delaquis, P.; Rempel, H.; Bach, S.; Harlton, C.; Aslam, M.; Pritchard, J.; Topp, E. Antibiotic resistance and diversity of Salmonella enterica serovars associated with broiler chickens. J. Food Prot. 2014, 77, 40-49.

34. Bankevich, A.; Nurk, S.; Antipov, D.; Gurevich, A.A.; Dvorkin, M.; Kulikov, A.S.; Lesin, V.M.; Nikolenko, S.I.; Pham, S.; Prjibelski, A.D.; et al. SPAdes: A new genome assembly algorithm and its applications to single-cell sequencing. J. Comput. Biol. 2012, 19, 455-477.

35. Halatsi, K.; Oikonomou, I.; Lambiri, M.; Mandilara, G.; Vatopoulos, A.; Kyriacou, A. PCR detection of Salmonella spp. using primers targeting the quorum sensing gene sdiA. FEMS Microbiol. Lett. 2006, 259, 201-207.

36. Ondov, B.D.; Treangen, T.J.; Melsted, P.; Mallonee, A.B.; Bergman, N.H.; Koren, S.; Phillippy, A.M. Mash: Fast genome and metagenome distance estimation using MinHash. Genome Biol. 2016, 17, 132.

37. Gurevich, A.; Saveliev, V.; Vyahhi, N.; Tesler, G. QUAST: Quality assessment tool for genome assemblies. Bioinformatics 2013, 29, 1072-1075.

38. Zhang, S.; Yin, Y.; Jones, M.B.; Zhang, Z.; Deatherage Kaiser, B.L.; Dinsmore, B.A.; Fitzgerald, C.; Fields, P.I.; Deng, X. Salmonella serotype determination utilizing high-throughput genome sequencing data. J. Clin. Microbiol. 2015, 53, $1685-1692$.

39. Zankari, E.; Hasman, H.; Cosentino, S.; Vestergaard, M.; Rasmussen, S.; Lund, O.; Aarestrup, F.M.; Larsen, M.V. Identification of acquired antimicrobial resistance genes. J. Antimicrob. Chemother 2012, 67, 2640-2644.

40. Brettin, T.; Davis, J.J.; Disz, T.; Edwards, R.A.; Gerdes, S.; Olsen, G.J.; Olson, R.; Overbeek, R.; Parrello, B.; Pusch, G.D.; et al. RASTtk: A modular and extensible implementation of the RAST algorithm for building custom annotation pipelines and annotating batches of genomes. Sci. Rep. 2015, 5, 8365.

41. Wattam, A.R.; Davis, J.J.; Assaf, R.; Boisvert, S.; Brettin, T.; Bun, C.; Conrad, N.; Dietrich, E.M.; Disz, T.; Gabbard, J.L.; et al. Improvements to PATRIC, the all-bacterial Bioinformatics Database and Analysis Resource Center. Nucleic. Acids. Res. 2017, 45, D535-D542.

42. Patchanee, P.; Eiamsam-Ang, T.; Vanaseang, J.; Boonkhot, P.; Tadee, P. Determination of regional relationships among Salmonella spp. isolated from retail pork circulating in the Chiang Mai municipality area using a WGS data approach. Int. J. Food Microbiol. 2017, 254, 18-24.

43. Davis, S.P.J.; Luo, Y.; Payne, J.; Shpuntoff, A.; Rand, H.; Strain, E. CFSAN SNP Pipeline: An automated method for constructing SNP matrices from next-generation sequence data. PeerJ. Computer Sci. 2015, 1, e20.

44. Guindon, S.; Dufayard, J.F.; Lefort, V.; Anisimova, M.; Hordijk, W.; Gascuel, O. New algorithms and methods to estimate maximum-likelihood phylogenies: Assessing the performance of PhyML 3.0. Syst. Biol. 2010, 59, 307-321.

45. Zhou, Z.; Alikhan, N.F.; Sergeant, M.J.; Luhmann, N.; Vaz, C.; Francisco, A.P.; Carrico, J.A.; Achtman, M. GrapeTree: Visualization of core genomic relationships among 100,000 bacterial pathogens. Genome. Res. 2018, 28, 1395-1404. 